\title{
A Systems-Based Dissection of Retinal Inputs to the Zebrafish Tectum Reveals Different Rules for Different Functional Classes during Development
}

\author{
Andrew S. Lowe, Nikolas Nikolaou, Paul R. Hunter, Ian D. Thompson, and Martin P. Meyer \\ MRC Centre for Developmental Neurobiology, King's College London, Guy's Hospital Campus, London SE1 1UL, United Kingdom
}

We have examined the form, diversity, and organization of three functional classes of retinal inputs to the zebrafish optic tectum during development. Our systems-based approach was to analyze data from populations of retinal ganglion cells labeled with a presynaptic targeted calcium indicator, synaptophysin GCaMP3 (SyGCaMP3). Collectively, our findings provide an insight as to the degree of visual encoding during retino-tectal development and how it dynamically evolves from a nascent and noisy presynaptic neural-scape to an increasingly complex and refined representation. We report five key features: (1) direction-selective inputs are developmentally invariant; (2) orientation-selective inputs exhibit highly dynamic properties over the same period, with changes in their functional characteristics and spatial organization; (3) inputs defined as anisotropic are an early dominant functional class, with heterogeneous response profiles, which progressively diminish in incidence and spatial extent; (4) dark rearing selectively affects the orientation-selective responses: both functional characteristics and relative spatial distributions; and (5) orientation-selective inputs exhibit four subtypes, two more than previously identified in any species. Our approach was to label RGC axon terminals with an indicator of activity and quantitatively characterize coherent response properties to different visual stimuli. Its application in the zebrafish, given its small size and the accessibility of the tectum, has enabled a quick yet robust assessment of multiple functional populations of responses.

\section{Introduction}

A major challenge in neuroscience is to understand how ensembles of neurons collectively organize and function within local circuits. Studying the anatomical and functional properties of individual cells on a cell-by-cell basis may well be an overly reductionist approach in this endeavor (Alivisatos et al., 2012; Carandini, 2012). The zebrafish is an emerging model system within neuroscience (Engert, 2013) that enables more holistic approaches. The zebrafish larva is small, transparent, and easily genetically engineered (Wyart and Del Bene, 2011), attributes that have enabled assessments of brain-wide neural activity during virtual-world paradigms (Ahrens et al., 2012) and complex visual processing (Ramdya and Engert, 2008; Gabriel et al., 2012; Grama and Engert, 2012; Nikolaou et al., 2012). Here we report a systems-based examination of the functional classes of retinal

Received May 2, 2013; revised June 25, 2013; accepted July 20, 2013.

Author contributions: A.S.L., N.N., P.R.H., I.D.T., and M.P.M. designed research; N.N. performed research; A.S.L. and P.R.H. contributed unpublished reagents/analytic tools; A.S.L. analyzed data; A.S.L., N.N., P.R.H., I.D.T., and M.P.M. wrote the paper.

A.S.L. is supported by Wellcome Trust program Grant 083205 (awarded to I.D.T.). N.N., P.R.H., and M.P.M. are supported by MRC project grants and a Career Development Award to M.P.M. (G0801242, G1100162, and G0600107). We thank Stephen Eglen, Samuel Barnes, and Juan Burrone for comments on the manuscript and Koichi Kawakami for Tol2 reagents.

The authors declare no competing financial interests.

Correspondence should be addressed to either Dr. Ian D. Thompson or Dr. Martin P. Meyer, MRC Centre for Developmental Neurobiology, King's College London, Guy's Hospital Campus, London SE1 1UL, United Kingdom, E-mail: ian.thompson@kcl.ac.uk or martin.meyer@kcl.ac.uk.

DOI:10.1523/JNEUROSCI.1866-13.2013

Copyright $\odot 2013$ the authors $\quad 0270-6474 / 13 / 3313946-11 \$ 15.00 / 0$ ganglion cells (RGCs), imaged in vivo and en masse as inputs to the zebrafish tectum.

The optic tectum, the primary retino-recipient brain region in the zebrafish, has a critical role in complex visually guided behaviors (Gahtan et al., 2005; Nevin et al., 2008; Fero et al., 2011). Its retino-recipient organization is stereotyped, with individual RGC axons being restricted to a single lamina (Xiao and Baier, 2007; Nevin et al., 2008; Robles et al., 2013), with an individual lamina populated by multiple classes of RGCs (Robles et al., 2013). However, very little is known about the functional characteristics of such visual inputs to the tectum. Indeed, given the zebrafish's very rapid development and that it is visually interactive before both retinal and tectal circuits are mature, there is a critical lack of information concerning the form, diversity, and organization of functional RGC inputs during the most significant period of retino-tectal development: 3-10 d postfertilization (dpf).

We have used a genetically targeted calcium indicator, specifically expressed in RGC axonal terminals (synaptophysin GCaMP3, SyGCaMP3) (Nikolaou et al., 2012), to dissect the functional inputs to the zebrafish larval optic tectum during development. A central tenet of this study was to examine whether population codes could be extracted using a multiple-neuron, single-trial approach rather than the more classical multiple-trial, single-neuron methods. Indeed, further, we extended that framework into a multiple-neuron, multiple-fish approach to build group parametric maps that represent the spatial organization of RGC inputs within a standard tectal neuropil. Our approach has revealed a complex picture of visual inputs in terms of their func- 
tional characteristics and spatial organization within the neuropil. Collectively, our findings provide a unique insight as to the degree of visual encoding during retino-tectal development.

\section{Materials and Methods}

\section{Animals}

Zebrafish (of indeterminate sex) were maintained at $28.5^{\circ} \mathrm{C}$ on either a $14 \mathrm{~h}$ ON/10 h OFF light cycle or for dark-reared larvae perpetual $24 \mathrm{~h}$ OFF initiated $\sim 6-10 \mathrm{~h}$ postfertilization. Transgenic lines used in this study include $\mathrm{Tg}(\mathrm{Isl} 2 \mathrm{~b}$ :Gal4) (Ben Fredj et al., 2010) and $\mathrm{Tg}$ (UAS:SyGCaMP3) as per Nikolaou et al. (2012). All experiments were performed in the pigmentation mutant, nacre, which lacks all neural crest-derived melanophores (Lister et al., 1999). This work was approved by the local Animal Care and Use Committee (King's College London) and was performed in accordance with the Animals Experimental Procedures Act, 1986, under license from the United Kingdom Home Office. Three ages of larvae were studied: $3 \mathrm{dpf}(n=5), 7 \mathrm{dpf}$ (normal rearing, $n=7$; dark rearing, $n=5)$, and $10 \mathrm{dpf}(n=9)$. Dark rearing was not attempted beyond $7 \mathrm{dpf}$ as the larval yolk sac has been virtually depleted by this time and larvae need to feed to survive.

\section{In vivo imaging and visual stimulus presentation}

Nonanesthetized $\mathrm{Tg}$ (Isl2b:Gal4)xTg(UAS:SyGCaMP3) larvae were immobilized in 2\% low melting point agarose (Sigma-Aldrich) prepared in Danieau solution and mounted dorsal side up on a raised glass platform that was placed in a custom-made Danieau-filled chamber. The agarose was sufficient to restrain the larvae so that anesthesia was not required. A diffusive filter 3026 (Rosco) was bonded to one side of the chamber to serve as a projection screen. The agarose in front of the eye facing the projection screen was removed, allowing an unobstructed view of the projected image on the side of the chamber while permitting the objective to be positioned above the tectum for imaging (Fig. 1A). Larvae were positioned $3 \mathrm{~cm}$ away from the screen, and the projected image filled a visual field of $\sim 97^{\circ} \times 63^{\circ}$ (Fig. $1 A$ ).

Visual stimuli consisted of light $\left(56 \mathrm{~cd} / \mathrm{m}^{2}\right)$ or dark bars $\left(8 \mathrm{~cd} / \mathrm{m}^{2}\right)$ ( $175 \%$ and $25 \%$ of mean, respectively) on a mean gray background ( 32 $\left.\mathrm{cd} / \mathrm{m}^{2}\right)$. As no qualitative differences between light and dark bars were noted, data for the two stimuli are combined. Each bar was $10^{\circ}$ in width moving at a speed of $20^{\circ}$ s and separated from the preceding bar by $30^{\circ}$, enabling more than one bar on the screen at any one time. The long axis of the bar was orthogonal to the direction of motion. A drifting bar stimulus has been used previously to define direction and orientation selectivity in the nonmammalian visual system (Bilotta and Abramov, 1989; Engert et al., 2002; Nikolaou et al., 2012). Visual experiments were generated and controlled using custom-written Labview and MATLAB code (MathWorks), implemented on a ViSaGe stimulus presenter (Cambridge Research Systems), and delivered via a DLP pico projector (Optoma).

Confocal imaging of visually evoked SyGCaMP3 responses was performed using an LSM 710 confocal microscope equipped with a spectral detection scan head and a 20×/1.0 NA water-immersion objective (Carl Zeiss). Functional time-series were acquired at a rate of $4.1 \mathrm{~Hz}$ and $0.415 \times 0.415 \mu \mathrm{m}$ resolution $(256 \times 256$ pixels $)$ and 1 A.U. pinhole aperture ( $\cong 1.4 \mu \mathrm{m}$ in $z$-axis) at three depths separated by $2 \mu \mathrm{m}$. The average diameter of a presynaptic bouton in zebrafish RGCs is $\sim 0.8 \mu \mathrm{m}$ (Meyer and Smith, 2006). Thus, the physical X-Y dimensions of voxels are below that of a typical presynaptic bouton. Excitation was provided by $488 \mathrm{~nm}$ multiline laser. Occasionally, we saw responses in isolated voxels at the onset of the laser, but generally no responses were observed unless visual stimuli were applied. Each orientation was presented once $(3 \mathrm{~s})$ in a pseudo-random order unique to each slice in every animal imaged; each interepoch interval was 20 s to enable SyGCaMP3 signals to return baseline and provide enough baseline to determine the knot for cublic spline interpolation of the baseline signals (see Voxelwise analysis). A blank-screen null condition of $2 \mathrm{~s}$ was also interleaved. A 3D stack of the entire tectal neuropil was acquired for each larva imaged to correct for pitch differences (and thus viewing orientation differences) between animals (see Pitch correction).

\section{Pitch correction}

To correct for differences in viewing orientations, a correction factor was determined from the relative pitch of animals within each age group. Pitch correction was limited to within age groups as larvae undergo substantial growth and axis rotations between 3, 7, and $10 \mathrm{dpf}$; thus, the exact relationship between the tectal neuropil and the eye was not known. To estimate the pitch differences between animals within each age group, the 3D stacks of the tectal neuropil were aligned to each other (spm8; www.fil.ion.ucl.ac.uk/spm), a process that reveals the degree of roll, yaw, and pitch rotations required to align all neuropils. The correction angle was applied to the calculated preferred direction or orientation of motion.

\section{Voxelwise analysis}

Preprocessing. The confocal time-series was postprocessed before analysis as follows: time-series images from each experiment were corrected for motion with a rigid-body algorithm (spm8; www.fil.ion.ucl.ac.uk/spm; see Fig. $1 C$ for an example voxel), median filtered with a kernel size of 1 voxel $(0.415 \mu \mathrm{m})$ to remove dark and shot noise, and spatially smoothed with a $2 \mathrm{D}$ Gaussian kernel $=2$ voxels $(0.83 \mu \mathrm{m})$ to improve signal-tonoise. A baseline (B) that corrects for low-frequency drifts was determined using a cubic-spline algorithm extrapolating between knots averaged from $5 \mathrm{~s}$ of the interepoch-interval data ( $\sim 20$ time-frames). Reference anatomical images were derived from the average of images at this stage of postprocessing. Relative signal intensity changes $(\Delta \mathrm{F}=\mathrm{F}-$ $\mathrm{B}$; where $\mathrm{F}=$ raw fluorescence) were calculated at each voxel and the integral response over the epoch-interval calculated to provide a single response metric of each presented orientation (see Fig. $1 C$ for an example voxel). There were relative signal changes $(\Delta \mathrm{F})$ as opposed to a normalized signal change $(\% \Delta \mathrm{F} / \mathrm{F} \equiv(\mathrm{F}-\mathrm{B}) / \mathrm{B})$ as we were only interested in within voxel signal changes rather than between voxel comparisons. Further, $\Delta \mathrm{F}$ has better contrast-to-noise ratio than $\% \Delta \mathrm{F} / \mathrm{F}$. The integral within each epoch window is a summary metric more resistant to saturation effects of the calcium probe than maximum signal change (Nikolaou et al., 2012).

Visually reactive voxels and metrics of visual response. A threshold for each voxel within an acquisition image sequence was determined from the variance of $\Delta \mathrm{F}$ changes during the interepoch intervals and null condition, threshold $\equiv 5 \times$ SDs. All voxels that were suprathreshold within at least two visually presentation epochs were regarded as visually responsive and subjected to further characterization: direction selectivity, orientation selectivity, and anisotropy. Direction- and orientationselective indices (DSI and OSI) (Niell and Stryker, 2008), based on fitted von-Mises profiles (Swindale, 1998), were calculated together with an estimate for their goodness of fit, $R^{2}$. To minimize cross talk and overfitting associated with DSI and OSI metrics, a stringent approach was undertaken. For a voxel to be regarded as DS or OS, mutually exclusive criteria were used: DS if DSI $>0.5$ and OSI $<0.5$; and OS if OSI $>0.5$ and DSI $<0.5$ (see Fig. $1 B$ ). In both cases, the goodness of fit for DSI and OSI, respectively, had to be $>0.8$; thus, the fitted curves explained at least $80 \%$ of the integral responses. The fitted curves used to estimate DSI and OSI also provided an estimate of the preferred direction or orientation of motion, respectively, from the center of the fitted curve. A pitch correction factor was applied within individual age groups to correct for differences in the viewing orientation of individual animals. For anisotropic responses, a $\chi^{2}$ value was calculated at each visually responsive voxel and those with a statistically significant value $(\mathrm{df}=1,11 ; p<0.05)$ were regarded as anisotropic (Sernagor and Grzywacz, 1995) (Fig. 1B). In essence, the $\chi^{2}$ metric identifies voxels with an unusually high variance for which some DS- and OS-like responses will also be included and so anisotropy is not mutually exclusive from the DS and OS responses (see next paragraph, Wavelet decomposition and cluster analysis, for their subsequent removal).

Wavelet decomposition and cluster analysis. Voxels identified as DS, OS, and/or anisotropic were further analyzed to assess whether there were coherent subtypes of responses across animals within each age/condition group. The integral responses to each presented oriented moving bar were standardized to eliminate the influence of different absolute degrees of response (zero mean and unit variance), giving each voxel approxi- 
A

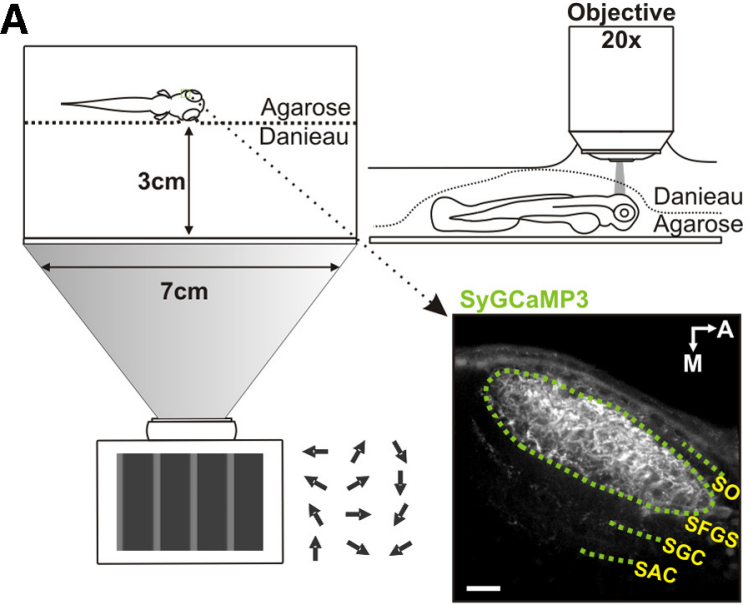

C
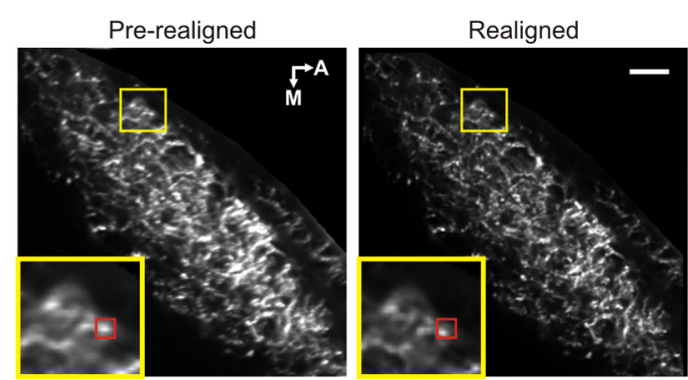

ii

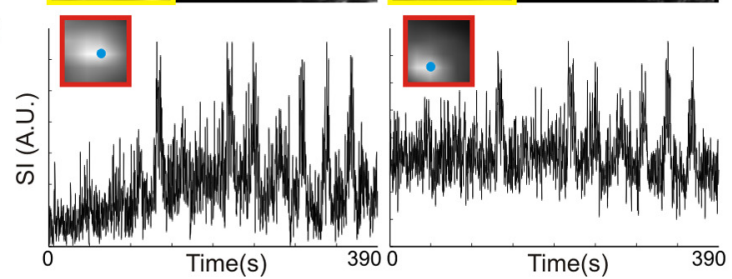

iii

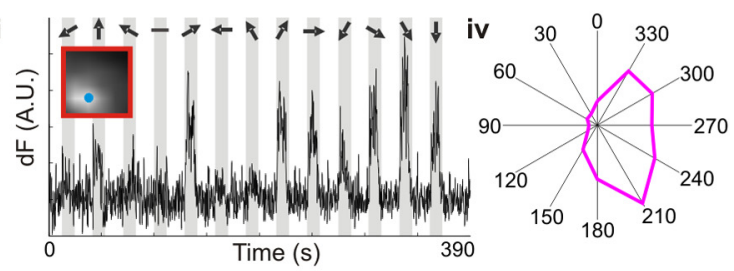

E

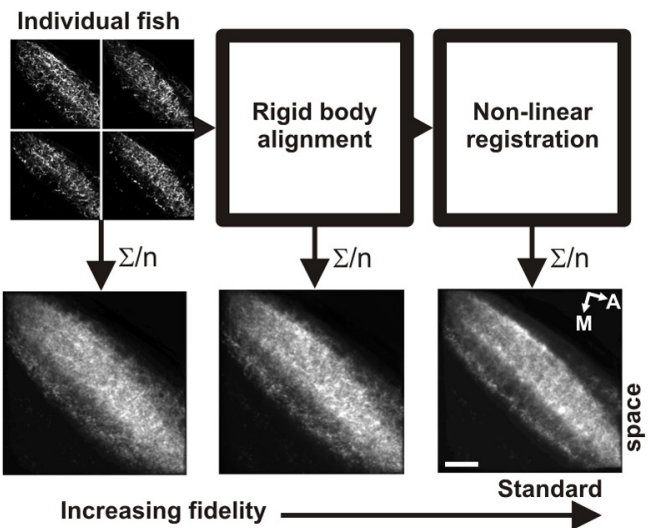

B

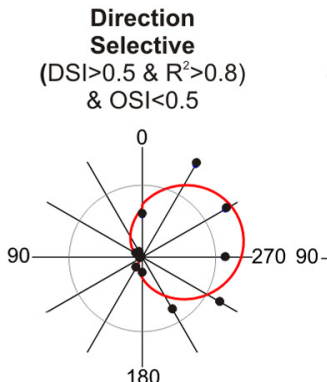

180

$\mathrm{DS} \mid=0.97$

OSI $=0.48$

$\mathrm{R}^{2}=0.91$

Typical

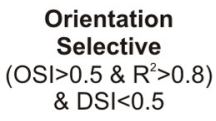

Orientation Selective (OSI>0.5\& $\left.R^{2}>0.8\right)$ $\& \mathrm{DSI}<0.5$

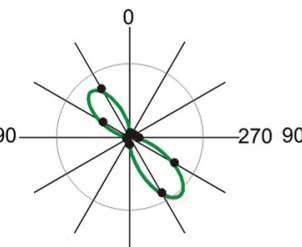

180

\section{Anisotropic}

$\mathrm{Chi}^{2}$

$d f=1,11 ; p<0.05$
D

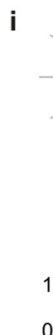

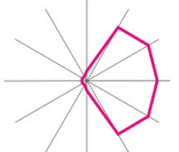

Feature
Preserving
Normalisation

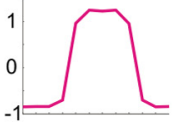

OSI $=0.99$
$D S I=0.12$
$R^{2}=0.96$
Typical

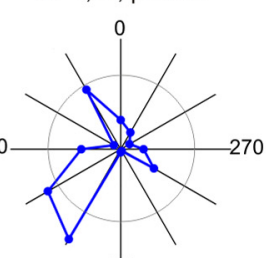

180

$\mathrm{Chi}^{2}=21.5$

$\mathrm{p}=0.03$

Example ii

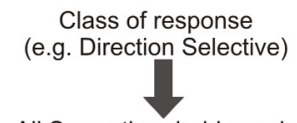

All Supra-threshold voxels

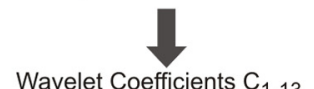

$\mathrm{D}_{2}$

iii

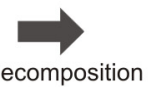

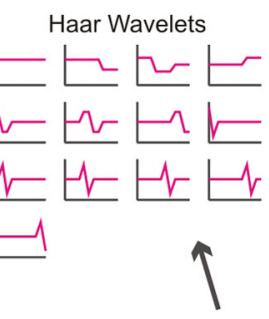

${ }_{i=1}^{13}$ Coefficients $_{i} \times$ Wavelets $_{i}$<smiles>[2H][Mg]</smiles>

$\mathrm{D}_{2}$

$\mathrm{D}_{3}$

$\mathrm{D}_{4}$

$\mathrm{D}_{5}$<smiles>O</smiles><smiles>[CH+]1[CH+]CC1</smiles>
난

$D_{2}$
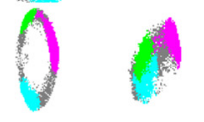

Wavelet Coefficients $\mathrm{C}_{1-13}$

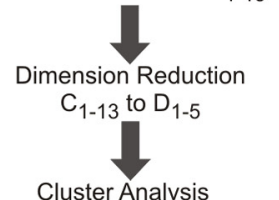

$\mathrm{D}_{3}$

$\mathrm{D}_{4}$

F

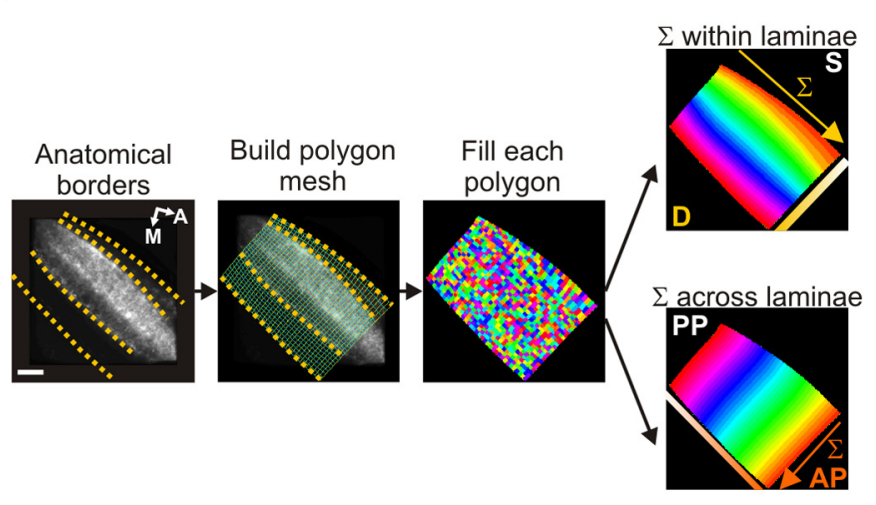

Figure 1. Schema for experimental setup, quantification, and data analysis. $A$, Nonanesthetized larvae, immobilized in $2 \%$ agarose, viewed projected moving bars (12 different, pseudorandomly ordered, orientations). Visually evoked SyGCaMP3 responses within RGC axon terminals were recorded in the contralateral tectum (inset expanded panel) using confocal imaging. SO, Stratum opticum; SGC, stratum griseum central; $S A C$, stratum album central. $B$, Three response characteristics to the oriented moving bars were explored: DS, 0 , and anisotropy. Polar plots of integral responses for individual voxels within each class. To minimize cross talk between the DS and OS response classes, stringent mutually exclusive criteria were used. In addition, a goodness of fit $\left(R^{2}\right)$ associated with each fitted curve, used to derive DSI and OSI, minimized overfitting. Anisotropic responses were determined according to their $\chi^{2}$ value. $C$, An example voxel precorrection and postcorrection for motion. Ci, Mean images across the time-series. Inset panels, Representative improvement after realignment. Red box is in the same position in both (Figure legend continues.) 
mately equal weighting. The normalized responses (Fig. 1Di) were decomposed into a sum of scaled (coefficients in Fig. 1Di) orthonormal Haar wavelets using in-house MATLAB (MathWorks) scripts. Wavelet decomposition is analogous to Fourier decomposition in that they both decompose an original signal into its primitive or fundamental constituents. Wavelets have the advantage over Fouriercomponents as they enable the location of a specific constituent (wavelet) to be determined. Examining the variance of the wavelet decomposition vectors within each class of response (DS, OS, and anisotropy) revealed those wavelets that account for the greatest variation of response profiles (Fig. 1Dii). Restricting further analysis to the most variant wavelet decomposition vectors has two benefits: (1) it provides a framework for optimal delineation of different responses; and (2) it reduces the dimension of response space, which improves reliability to resolve independent response subtypes. The five most variable decomposition vectors (highest variance within group) were regarded as a 5-D space of responses and examined for clusters that would be indicative of coherent responses (D1-D5, Fig. 1Diii). A spike sorting software was modified in-house to examine the integral wavelet response space (wave_clus; http://www2.le.ac.uk/ departments/engineering/research/bioengineering/neuroengineering-lab/spikesorting) (Quiroga et al., 2004). The software is unsupervised but allows for (1) the manual exclusion of "unlikely" clusters of responses, identified clusters with incoherent or mixed response profiles and (2) the "forcing" of unclustered responses into a nearby cluster. All identified clusters were confirmed by manually examining (1) the mean raw response profile for all voxels within a cluster to ensure a univariate rather than multivariate response profile and (2) projections in wavelet space (Fig. 1Diii) to ensure the coherence and separation between identified clusters. DS and OS voxels were examined separately and a number of clusters with coherent response profiles identified with a smaller fraction of voxels failing to cluster, called residuals. These residuals may represent measurement errors, false-positives (incorrectly attributed to a response class), or transition voxels. In contrast, the anisotropic voxels generally failed to cluster, although a limited number of clusters were identified that exhibited DS- or OS-like features. These clusters were regarded as coherent anisotropic responses and excluded from the anisotropic class. All anisotropic responses reported are those that failed to cluster being regarded as incoherent responses.

Cumulative histograms. Grouped cumulative histograms of the preferred direction or orientation of motion were constructed from the calculated preferred angles ( + within age correction for pitch) for each identified cluster of responses in wavelet space. Each cluster exhibited a normal distribution of preferred angles (data not shown) that is indicative of a uniformly distributed responses. Each distribution of preferred angles for each cluster was fitted by a von Mises distribution using a multidimensional constrained nonlinear minimization, with peak center, height, concentration, and base height being free dimensions.

Constructing standard tectal neuropil space. An atlas image for each functional imaging time-series was produced from the estimated baseline responses (Fig. 1B), thus producing an anatomical image unbiased by relative degrees of visual reactivity. A standard tectal space for each age group was constructed using a two step process: rigid body alignment and nonlinear registration (Fig. 1C). First, a single random animal was chosen for rigid-body alignment (deformation free) of all imaged slices across all animals. Averaging across all realigned atlas images produced a first template image for subsequent nonlinear registration-free form deformation using a block matching approach (Ourselin et al., 2001) (http://cmic.cs.ucl.ac.uk/home/software/). After nonlinear registration, a new template was created and the rigid-body aligned data reregistered. This iterative process was repeated 3 times, producing a high-fidelity

\section{$\leftarrow$}

(Figure legend continued.) images. Cii, Raw data for a single voxel, marked by blue dot inside red inset panel. Ciii, After preprocessing. Civ, Polar plot of integral values. D, Schemata of wavelet decomposition and cluster analyses. Di, Single voxel. Integral values are normalized and decomposed. Dii, Mulitple voxels within a functional class are clustered into subtypes. Diii, Example of clustered DS subtypes. $\boldsymbol{E}$, Formation of standard neuropil space by a two stageprocess. $\boldsymbol{F}$, Formation of polygon space to collate responsive voxels across and within laminae. Scale bars, $20 \mu \mathrm{m}$. template image (Fig. 1C) for each age group that subsequently improved the registration process. Parametric maps of each metric (DS, OS, and anisotropy) were placed into standard space using the affine transformations from the rigid-body alignment plus the free-form nonlinear blockwise transformations.

Polygon space. To account for the curved nature of the tectum, we constructed a curved polygon mesh to each neuropil standard space. A manual delineation of anatomically distinct boundaries (borders of SO, stratum fibrosum et griseum superficiale [SFGS], and SGS) on each standard space image enabled a polygon mesh to be generated using an in-house MATLAB script (MathWorks) (Fig. 1D). Applying the polygon mesh to the individual parametric maps and summing the incidence of indentified voxels along the axes of the polygon mesh effectively provides a linear profile for each axis.

\section{Results}

\section{Dark rearing reveals differential susceptibilities across response classes}

Functional imaging of RGC terminals within the zebrafish larval tectum reveals three subtypes of DS and four subtypes of OS responses at $7 \mathrm{dpf}$, with $(n=5)$ or without dark rearing $(n=7)$ (Fig. 2A,B). Using a wavelet decomposition and model-free, semiautomatic cluster analysis approach provided an objective means of identifying and resolving coherent subtypes of responses within each class (DS and OS) (see polar plots of responses for each clustered subtype) (Fig. 2C,D; see Fig. 5). Figure $2 A, B$ (histograms) represent the cumulative incidence of voxels within each subtype for DS and OS responses, respectively, collated according to their calculated preferred directions or orientations of motion. Each identified subtype is color-coded, with gray representing voxels that failed to cluster. Under both normal and dark rearing conditions, the three subtypes of DS responses are consistent with those previously described at this age (Nikolaou et al., 2012), whereas the identification of four OS subtypes, compared with the two previously described in zebrafish (Nikolaou et al., 2012) or indeed any species (Levick, 1967; Sernagor and Grzywacz, 1995; Venkataramani and Taylor, 2010), emphasizes the significant improvement in sensitivity and specificity of our approach. Fitting distribution curves to the cumulative histogram of each subtype provided the peak preferred angle of motion (Fig. $2 A, B$, fitted black curves and pinwheel insets). Collectively, the DS subtypes appear unaltered by dark rearing with coherent cumulative distributions and peak preferred angles (Fig. 2A), whereas with the four OS subtypes, although still coherent, their peak preferred orientations are all shifted to the right (Fig. 2B).

Given a differential effect of dark rearing on the characteristics of the DS and OS functional classes, was their terminal-neuropil organization within the tectum similarly affected? To answer this question, an objective assessment of the spatial organization of responsive voxels was developed. All imaged fish were spatially registered into a common tectal space (Fig. 2E, gray underlay image) that was segmented by a polygon mesh that followed the curved tectal neuropil (Fig. 2E, overlaid mesh). The spatial distributions of each DS and OS subtype across multiple fish were collated by summing across orthogonal axes of these polygon meshes, providing information about the spatial location of classes of response across laminar or polar axes of the neuropil. Examining the distribution of the relative signal intensities of SyGCaMP3-expressing voxels (averaged across all fish imaged within the $7 \mathrm{dpf}$ normal- and dark-reared groups) across either axis suggests that there was no gross alteration in the expression of SyGCaMP3 associated with altered visual experience (Fig. 2F). Figure $2 G, H$ maps the cumulative spatial distributions of DS and 
A

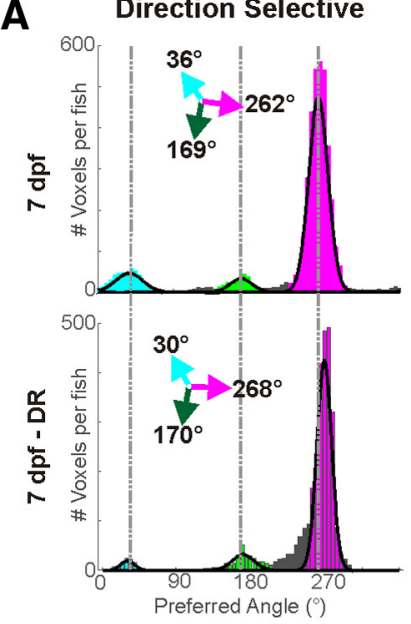

C Direction Selective

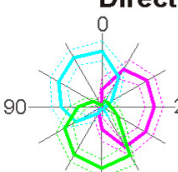

180

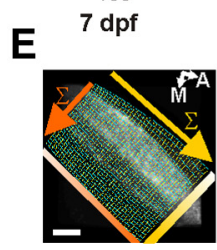

$7 \mathrm{dpf}$

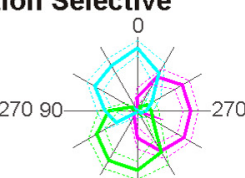

180

$7 \mathrm{dpf}$ - DR

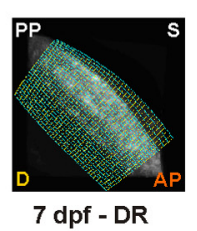

B

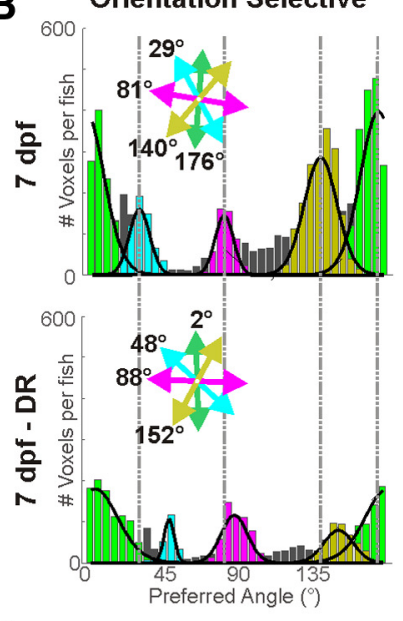

D

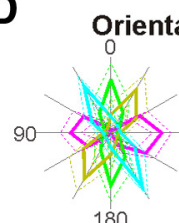

180

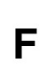

$\mathbf{F}$

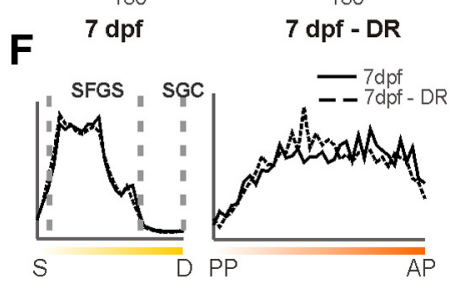

dpf - DR

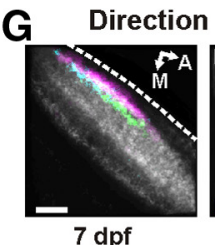

I

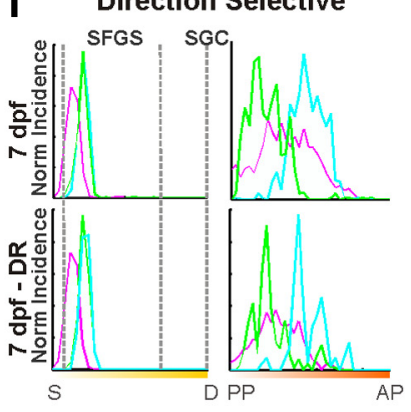

K

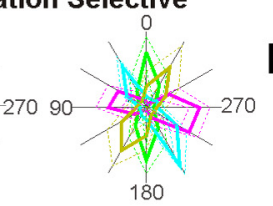

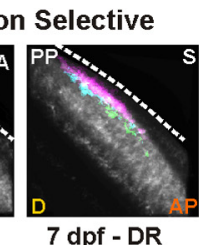

$7 \mathrm{dpf}$ - DR

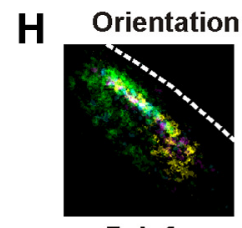

$7 \mathrm{dpf}$

J Orientation Selective

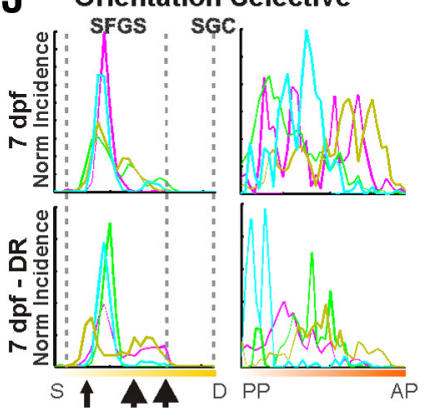

L

Orientation Selective

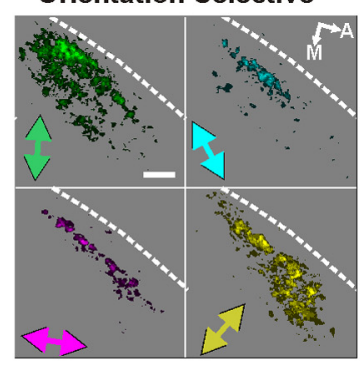

$7 \mathrm{dpf}$

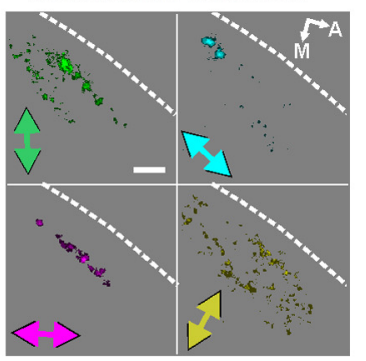

$7 \mathrm{dpf}$ - DR

Figure 2. Dark rearing, different rules for different classes. $A, B$, Cumulative histograms summarize the incidence of preferred angles for identified DS and OS voxels, respectively. Top panels, 7 $\operatorname{dpf}(n=7)$; bottom panels, $7 \mathrm{dpf}$ dark reared (DR) $(n=5)$. Individual subtypes of response identified by wavelet decomposition and cluster analysis of wavelet space are color-coded, with gray representing unclustered residuals. Overlaid curves are the fitted von Mises distributions for each identified DS $(\boldsymbol{A})$ or $0 S(\boldsymbol{B})$ subtype, with the color-coded arrow pinwheel representing individual peak preferred angles. Dotted lines are centered on the peak preferred angles at $7 \mathrm{dpf}$. C, D, Polar plots illustrating the mean ( $\pm 1 S D)$ normalized response profiles for each identified DS (C) and $0 S$ (D) subtypes within the $7 \mathrm{dpf}$ normal and dark-reared animals, color coding as per $\boldsymbol{A}$ and $\boldsymbol{B}$. E, Polygon meshes outline the tectal neuropil of $7 \mathrm{dpf}$ and $7 \mathrm{dpf}$ DR zebrafish. Each mesh spans the neuropil layers from SO to SGC (superficial to deep, S-D) and almost the entire posterior- to anterior-pole (PP-AP) axis. F, Line plots of the mean baseline signal across all fish imaged at $7 \mathrm{dpf}$ (solid line) and $7 \mathrm{dpf}$ DR (dashed line), illustrating the invariant anatomical organization of SyGCaMP3 labeled RGC axon terminals across groups. Left graph, Laminar organization. Vertical gray dotted lines are directly comparable anatomical boundaries. $\boldsymbol{G}, \boldsymbol{H}$, Composite grouped parametric maps across all fish imaged illustrate the spatial organization of all $(\boldsymbol{G})$ DS and $(\boldsymbol{H}) 0 S$ subtypes within each age group. DS subtypes are overlaid on the standard space template image derived for each group, providing a reference. White dotted lines indicate the edge of the tectal $S 0$ lamina. Within the individual parametric maps, voxel brightness is proportional to the summed incidence of each functional response across all fish imaged. $I, J$, Line plots illustrating the spatial organization of $D S$ and OS subtypes, respectively; color coding as per $\boldsymbol{A}$ and $\boldsymbol{B}$. I, Broadly, these line plots exhibit invariant features associated with DR. J, Laminar organization appears disrupted (arrows) after DR with qualitatively different distributions across neuropil poles (PP-AP). $\boldsymbol{K}, \boldsymbol{L}$, Group parametric maps show the spatial organization of individual 0 s subtypes for all fish imaged at $7 \mathrm{dpf}(\boldsymbol{K})$ and $7 \mathrm{dpf} D \mathrm{DR}$ (L). White dotted lines indicate the edge of the tectal $S 0$ lamina. Scale bars, $20 \mu \mathrm{m}$.

OS subtypes across all imaged animals, with and without dark rearing, both exhibiting complex but coherent distributions. Figure $2 I, J$ examines the spatial distribution across the tectal neuropil of each subtype and functional class. The terminal-neuropil organization of the three DS subtypes of response (compare with Fig. 2I) appears entirely conserved between the two conditions. There is no apparent difference in the laminar distribution of the subtypes or in their relative distribution across the polar axis of the tectum. In contrast, the distribution of the four subtypes of OS responses appears to be affected by dark rearing (compare with Fig. 2J). In normally reared fish at $7 \mathrm{dpf}$, all four subtypes can be found within a central lamina within SFGS, with three subtypes displaying additional deeper distributions. After dark rearing, although a central SFGS lamina can be seen, one subtype now shows a clear ectopic arrangement (Fig. 2J, yellow subtype, narrow arrow), and there is an alteration in the coherence of the subtype contributions within the deeper neuropil (Fig. 2J, broad arrows). These alterations do not represent abnormalities in a limited number of fish (data not shown). The distribution of OS subtypes within laminae (i.e., pole to pole) in normally reared fish is complex and difficult to quantify further, but there are apparent qualitative differences after dark rearing (Fig. 2J-L). Thus, although SyGCaMP3-expressing voxels, representing all classes of RGCs innervating the tectum, appeared unaffected by altered visual experience during development, a very limited functional subset of RGCs (OS class) exhibited subtle qualitative alterations in their relative spatial organization. Collectively, these observations suggest that different developmental rules apply to OS and DS classes of RGC inputs to zebrafish tectum. Are such differences also associated with divergent developmental profiles?

Dynamic OS responses during development

The functional response properties of retinal inputs to the larval zebrafish tectum, reared under normal light/dark conditions, have been examined at 3,7 , and $10 \mathrm{dpf}$. This age range represents 
A

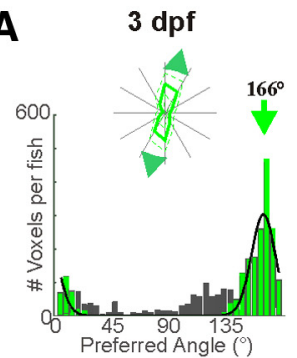

B

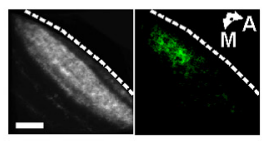

C

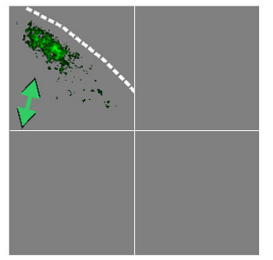

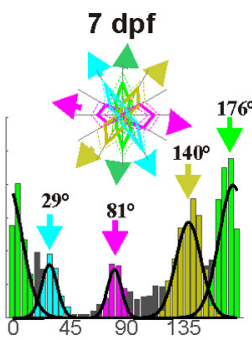
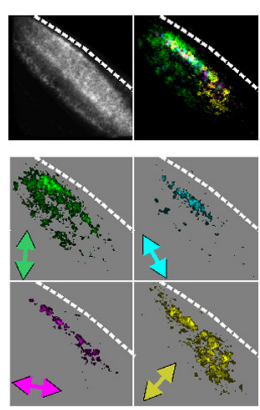
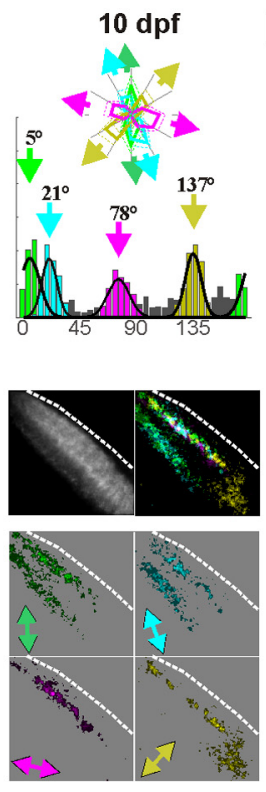

D

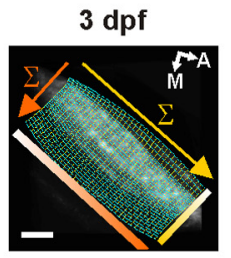

$7 \mathrm{dpf}$
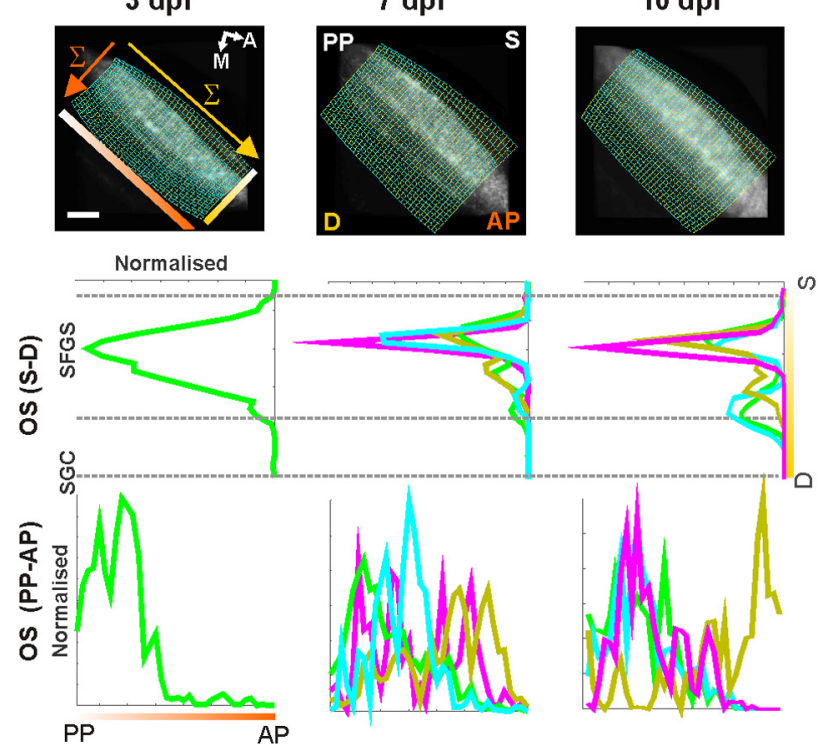

Figure 3. OS responses are highly dynamic during development. $A$, Cumulative histograms summarize the incidence of preferred angles for 05 voxels at 3,7 , and $10 \mathrm{dpf}$ ( $n=5,7$, and 9 , respectively). Individual subtypes of response identified by wavelet decomposition and cluster analysis of wavelet space are color-coded, with gray representing unclustered residuals. Overlaid curves are the fitted von Mises distributions for each identified OS subtype with the color-coded arrow pinwheel representing individual peak preferred angles. Inset, Polar plots of the mean (solid line) \pm 1 SD (dashed line) normalized integral responses to the presented bars, across all clustered voxels within each 0S subtype. $\boldsymbol{B}$, Composite grouped parametric maps across all fish imaged illustrate the spatial organization of all $0 S$ subtypes within each age group. The standard space template image derived for each group provides a reference, whereas the white dotted lines indicate the edge of the tectal SO lamina. Within the individual parametric maps, voxel brightness is proportional to the summed incidence of each functional response across all fish imaged. $\boldsymbol{C}$, Individual grouped parametric maps for each 0 S subtype within each age group. $\boldsymbol{D}$, Line plots illustrating the spatial organization of OS subtypes, respectively. Top, Standard tectal spaces and overlaid polygon meshes for each age group. Middle, Laminar organization. Bottom, Posterior-pole to anterior-pole spatial organization. Scale bars, $20 \mu \mathrm{m}$. Color coding in all cases, same as in $\boldsymbol{A}$.

the most significant period for retino-tectal development. By 3 $\mathrm{dpf}$, early retino-tectal synapse formation is just underway and simple visually mediated behaviors are evident (Kimmel et al., 1974; Easter and Nicola, 1996). Retino-tectal synaptic density has reached a plateau by $7 \mathrm{dpf}$ (Niell et al., 2004; Meyer and Smith, 2006), and larvae have accomplished, if stereotyped, visually mediated hunting behaviors (Fero et al., 2011). Within the second week of development (10 dpf), larvae exhibit increasingly adroit visually mediated behaviors as demonstrated by hints of social behaviors (Engeszer et al., 2007) while the retina is in a late phase of development (Mumm et al., 2006; Schroeter et al., 2006).

At $3 \mathrm{dpf}$, cluster analysis revealed only a single coherent OS subtype (Fig. $3 A ; \sim$ vertical motion, green) with diffuse residual nonclustered voxels evident in wavelet space (Fig. 3A; see Fig. 5). This OS subtype appears spatially restricted to the posterior pole of SFGS but broadly distributed across the laminar axis of SFGS (Fig. $3 A, C$ ). Figure $3 A$ (inset) shows the polar plots of normalized responses to the presented bars for all OS voxels at this age. As was seen in Figure 2, by 7 dpf, four subtypes were resolved, exhibiting varying degrees of diffuse, but broadly laminar, terminal-neuropil organization. By $10 \mathrm{dpf}$, four OS subtypes are still resolved, with independent normal distributions of preferred orientations (Fig. $3 A$ ) that individually (Fig. 3C) and collectively exhibited highly laminar distributions (Fig. $3 B-D$ ). These data reveal a very dynamic developmental picture of OS responses with an increasingly refined terminal-neuropil organization developing from 3-10 dpf.

There are specific differences of note between $7 \mathrm{dpf}$ and $10 \mathrm{dpf}$ both in relative tuning and laminar distributions. Two of the subtypes at $10 \mathrm{dpf}\left(\right.$ magenta, $\sim 78^{\circ}$; yellow, $\left.\sim 137^{\circ}\right)$ are similar in tuning profile and laminar distribution to two subtypes identified at $7 \mathrm{dpf}$. However, the other two subtypes (green, $\sim 5^{\circ}$; cyan, $\sim 21^{\circ}$ ) are more dynamic between these ages. They appear to be converging in their peak preferred orientations (Fig. $3 A$, overlapping populations) and refining into a clear bilaminar distribution within SFGS (Figure 3C,D). The polar plots of normalized responses to the presented bars (Fig. $3 A$, inset) confirm the convergence of the near-vertical subtypes at $10 \mathrm{dpf}$. There is no longer a clear and unique tiling of orientation space: one axis (vertical) is oversampled; and with the other two subtypes, there is now a triangular arrangement of preferred orientations.

It should be noted that, to reveal developmental dynamics, age/condition groups were analyzed independently; thus, the color coding of OS subtypes between 3 and $10 \mathrm{dpf}$ does not definitively reflect a coherence of subtypes across age groups. Color coding of OS subtypes at 7 and $10 \mathrm{dpf}$ was applied in the same order across the cumulative histogram, with the cluster closest to the OS subtype at $3 \mathrm{dpf}$ being color-coded as green. The coherent spatial distribution of subtypes across ages (Fig. $3 C, D$ ) provides supporting evidence that the clusters are appropriately assigned. It should also be noted that, although it is possible to correct for differences in the relative pitch of animals within an age group, it was not possible to correct across age groups. Thus, it was not possible to correct for relative eccentricities in how each age group actually viewed the presented stimuli.

\section{Developmentally invariant DS responses}

We have previously demonstrated three populations of DS inputs to the zebrafish tectum evident at $7 \mathrm{dpf}$, which were conspicuously organized in discrete laminae (Nikolaou et al., 2012). In this study, three DS subtypes are identified at each age: 3,7 , and $10 \mathrm{dpf}$ (Fig. 4; see Fig. 5). Within and across age groups, the identified DS subtypes consistently exhibit broad "flat-topped" response profiles (Fig. $4 A$, inset) that are more constrained by the band- 
width of response rather than a single dominant response, a hallmark of mammalian DS RGCs (Chan and Chiao, 2008; Elstrott et al., 2008; Huberman et al., 2009; Yonehara et al., 2009; Kim et al., 2010; Rivlin-Etzion et al., 2011). The broad response profiles of the three subtypes, at each age, collectively fill direction space, forming an approximately uniform triangular arrangement of relative preferred directions of motion (Fig. $4 A$, inset). Further, the three DS subtypes at each age exhibit a discrete laminar organization with the most abundant subtype $\left(\sim 270^{\circ}\right.$, magenta) uniquely found in a superficial lamina, whereas the other two subtypes occupy a common but slightly deeper lamina (Fig. 4B,C). Two of the three populations of DS subtypes at 3 and $10 \mathrm{dpf}$ are more sparsely evident than at 7 dpf, which may reflect differences in image acquisition planes across ages. Similarly, the apparent shift in peak preferred directions of motion (Fig. 4A, pinwheels) may reflect different relative viewing orientations of the eye across age groups. The final phase of rotations that align the head (and eye) with the long axis of the body (Kimmel et al., 1995), the progressive depletion of the yolk-sac, and eccentricities in how fish were embedded in agarose probably all contribute to such apparent differences in absolute peak preferred directions of motion at different ages. The continuity of the three identified DS subtypes in terms of their relative response profiles, triangular tiling of direction space, and coherent laminar organization collectively suggest that these DS subtypes are invariant during development. This finding was consistent with their apparent invariance following $7 \mathrm{~d}$ of dark rearing.

\section{Anisotropy is a dynamic developmental feature of RGC responses}

DS and OS subtypes exhibited responses to the 12 directions of motion that were phenotypic across fish, as revealed by wavelet decomposition and cluster analysis. However, not all visually reactive voxels exhibited such simple unimodal or bimodal responses; instead, some voxels exhibited complex anisotropic or "spiky" responses. To examine whether such responses were consistent across animals, they were examined further. A high $\chi^{2}$ value for responses to the 12 drifting bars provided a metric of anisotropy (Sernagor and Grzywacz, 1995). The wavelet decomposition of highly anisotropic responses to moving bars and subsequent cluster analysis confirmed that, unlike the DS and OS responses, anisotropic responses fill wavelet space and have no coherent clusters, suggesting that there were no phenotypic response subtypes (Fig. 5). The incidence and spatial organization of anisotropic responses varied with age. For the earliest age group ( $3 \mathrm{dpf}$ ), anisotropic responses exhibited a coherent triple laminar arrangement that appears distinct from combined DS and OS responses at this age (Fig. 6A,B). At later
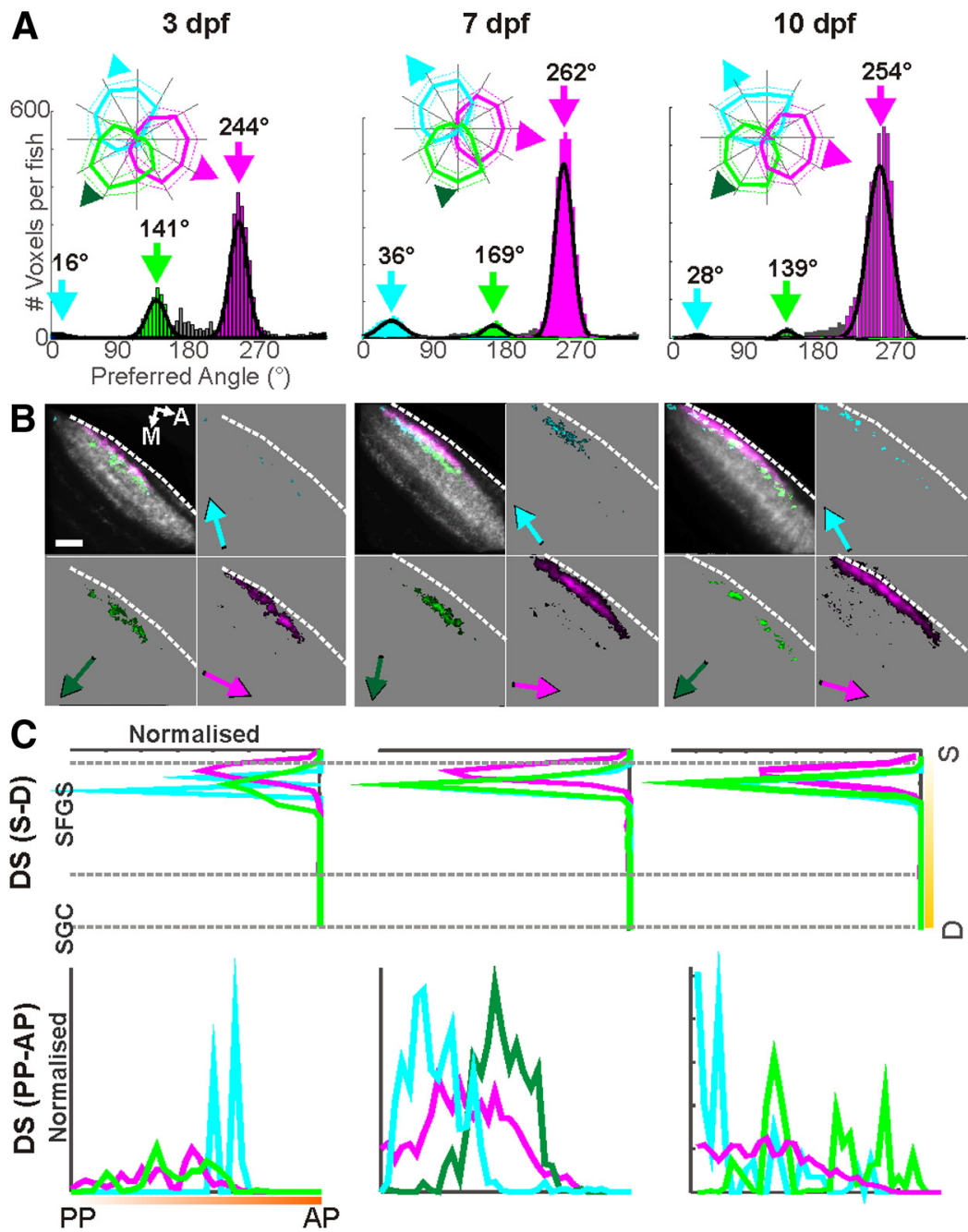

Figure 4. DS responses are invariant during development. $\boldsymbol{A}$, Cumulative histograms summarize the incidence of preferred angles for DS voxels at 3, 7, and $10 \mathrm{dpf}(n=5,7$, and 9, respectively). Individual subtypes of response identified by wavelet decomposition and cluster analysis of wavelet space are color-coded, with gray representing unclustered residuals. Overlaid curves fitted von Mises distributions for each identified DS subtype with the color-coded arrow pinwheel representing individual bars across all clustered voxels within each DS subtype. $\boldsymbol{B}$, Composite grouped parametric maps across all fish imaged spatial organization of all DS subtypes within each age group. The standard space template image derived for each organization of OS subtypes. Top, Laminar organization. Bottom, Posterior-pole to anterior-pole spatial organization. Scale bars, $20 \mu \mathrm{m}$. Color coding in all cases, same as in $\boldsymbol{A}$.

ages, these three laminae progressively refined, with only the most superficial laminar persisting to $10 \mathrm{dpf}$, being ultimately nestled between DS and OS laminae. While exhibiting a dynamic developmental profile, the spatial distribution of the anisotropic responses appeared unaffected by dark rearing (Fig. 6A,B); and although the incidence per fish was reduced, it was in line with comparable reductions in the incidence of DS and OS voxels (compare with Fig. 6B).

\section{Comparisons across classes}

Drifting bars have been used to identify three classes of response during development: OS, DS, and anisotropic responses. Figure 6 summarizes how the distribution of these classes changes during development, with all subtypes within each functional class being combined in to a single composite map in standard space (Fig. $6 A$ ). Collectively, DS responses are always located within the most superficial SFGS laminae, whereas the OS responses are 


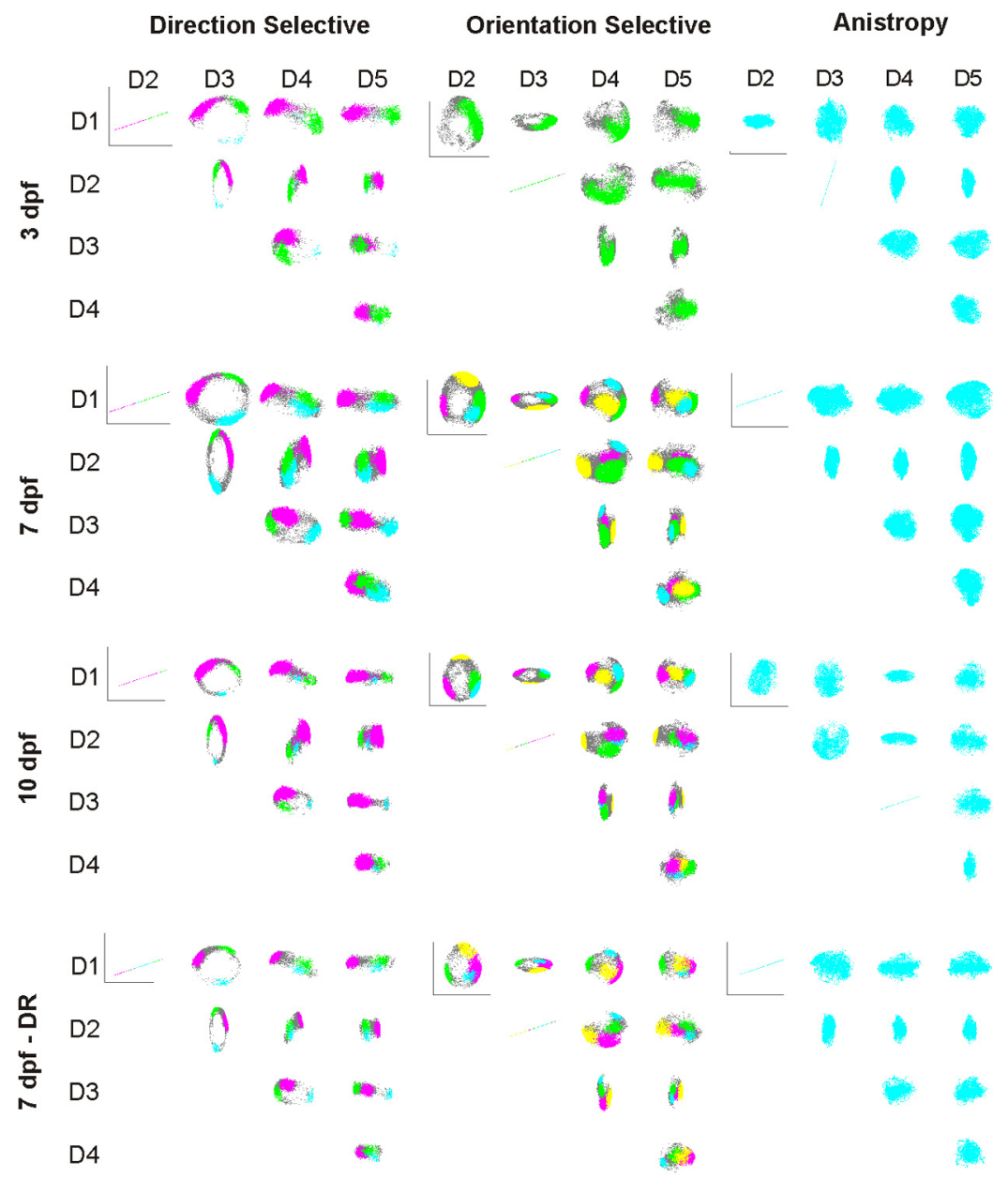

Figure 5. Identified clusters in wavelet space for the DS, 0 S, and anisotropic responses at 3, 7, and $10 \mathrm{dpf}$, and $7 \mathrm{dpf}$ after dark rearing. For the DS and $0 S$ responses, color-coded voxels represent identified clusters within wavelet space; and gray, the unclustered residuals. In contrast, anisotropic responses (cyan) fill wavelet space.

altogether more dynamic (Fig. 6A,B). Early in development, OS responses are broadly distributed across SFGS, although the peak is always deep to that of the DS responses. The OS distribution gradually refines to give a bilaminar distribution: the main peak in mid SFGS and a secondary peak in deeper SFGS. In complete contrast, anisotropic responses displayed a distinct trilaminar distribution at $3 \mathrm{dpf}$ that resolved to a single lamina in SFGS that is interposed between the DS subtypes and the most superficial OS lamina at $10 \mathrm{dpf}$. As the relative incidence of anisotropic responses decreases between 3 and $10 \mathrm{dpf}$ (Fig. 6C), it is tempting to speculate that some anisotropic responses may reflect developmental transients en route to other response classes. The persistence of a distinct superficial lamina at $10 \mathrm{dpf}$ suggests either different developmental dynamics or that anisotropy reflects a mature component of retinal processing, albeit one not optimally captured with drifting bars or Haar wavelet decomposition. Finally, although DS, OS, and anisotropic responses have been exhaustively examined, they only represent a relative small subset of voxels within the tectal neuropil that responded to drifting bars (Fig. 6D).

\section{Discussion}

Using a systems-based approach, we have been able to dissect the differential development of three functional classes of RGC input responses to the zebrafish tectum: DS, OS, and anisotropic. More specifically, we observe that DS inputs are developmentally invariant between 3 and $10 \mathrm{dpf}$ (in terms of their relative triangular response profiles, bandwidths, and laminar position); OS inputs exhibit highly dynamic properties over the same period, with changes in their relative functional characteristics and laminar distribution; inputs defined as anisotropic ("spiky" responses) are the early dominant class (3 dpf), which becomes both less common and more spatially restricted by $10 \mathrm{dpf}$; and dark rearing only affects the OS class of responses, both visual response characteristics and relative spatial distribution within the neuropil. Collectively, our findings provide unique insights as to the degree of visual encoding during retino-tectal development and how it dynamically evolves from a nascent and noisy presynaptic neural-scape to an increasingly complex and refined representation.

The number of zebrafish DS retinal subtypes and their preferred directions of motion match those of mammalian ON-DS ganglion cells (On-DSGCs) (Huberman et al., 2009; Yonehara et al., 2009; Vaney et al., 2012). The invariant developmental profile of the three identified subtypes reported here are directly comparable to the mammalian On-DSGCs, which are also invariant to altered visual experience and exhibit robust directional responses before and around the time of eye opening (Chan and Chiao, 2008; Elstrott et al., 2008). Although OS ganglion cells (OSGCs) have been reported in rabbits since the 1960s (Levick, 1967), this functional class of response has been studied little since. Indeed, although extensive work on DSGCs has revealed several subtypes in mammals (Vaney et al., 2012), OSGCs have only been observed for the two cardinal orientations of motion first proposed by Levick: vertical and horizontal (Levick, 1967; Sernagor and Grzywacz, 1995; Venkataramani and Taylor, 2010). We report four subtypes of OS response in RGC axon terminals that are highly dynamic during retino-tectal development. Indeed, such a dynamic profile suggests that visual experience may be a developmental driving force for this class of responses, which was confirmed by visual deprivation. To our knowledge, this is the first time that four OS-RGC subtypes have been reported in any species and that the functional characteristics of OS-RGCs are driven by visual experience during development.

The anisotropic response class is similarly dynamic during development in terms of incidence and laminar distribution, but there were no gross effects associated with dark rearing. The etiology of such anisotropic responses is not known, although their incoherence within wavelet space could be indicative of nonspecific (ectopic or noisy) responses to the stimuli, as previously reported in turtle embryos (Sernagor and Grzywacz, 1995), or they may exhibit coherent features that are not optimally deconvolved with Haar wavelets. The anisotropic responses reported within the zebrafish larval neuropil may reflect various develop- 
A

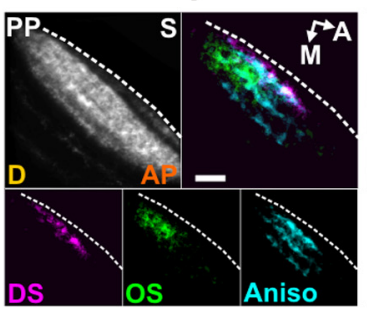

B
$7 \mathrm{dpf}$

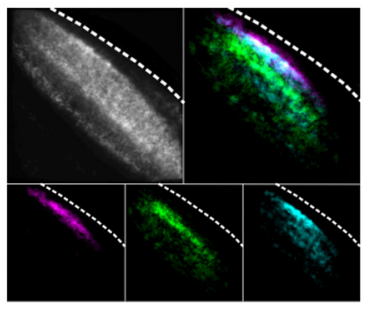

$10 \mathrm{dpf}$

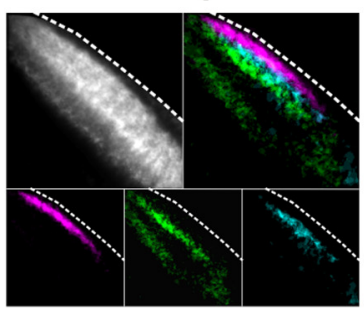

$7 \mathrm{dpf}$ - DR

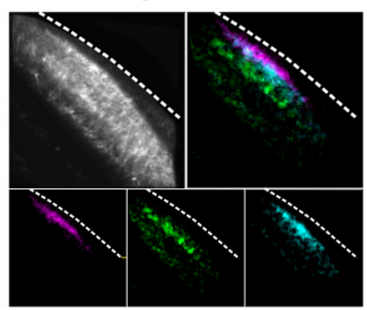

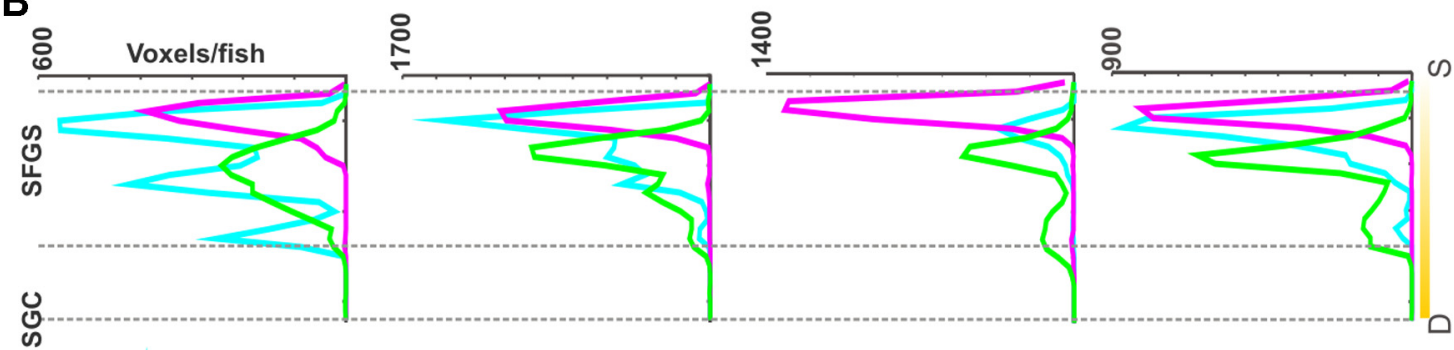
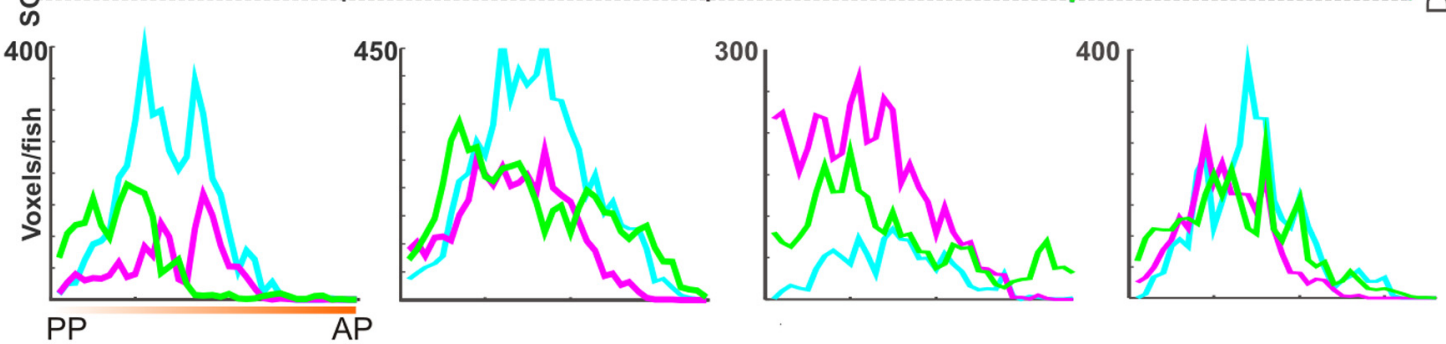

C
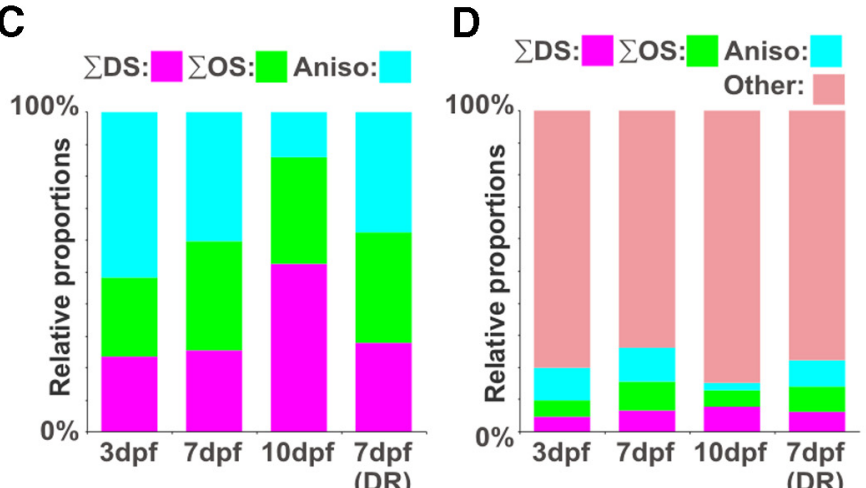

(DR)

Figure 6. Relative spatial organization of all $D S, O S$, and anisotropic responses. $A$, Grouped parametric maps across all fish imaged illustrate the relative spatial organization of all three classes of response: $D S, 0 S$, and anisotropy $\left(\chi^{2}\right)$ for each age group, 3, 7, and $10 \mathrm{dpf}$ and $7 \mathrm{dpf}$, DR. Top right panel within each age/condition group, Composite of all three classes of response, with individual color-coded panels for each class aiding comparison. Maps for DS and $0 S$ are derived from the summed incidence across all subtypes. White dotted lines indicate the edge of the tectal $S 0$ lamina. $\boldsymbol{B}$, Top, Averaged incidence (per fish) of voxels for each class of response across laminar (S-D axis). Dotted lines across line plots represent comparable boundaries of the SFGS lamina. Bottom, Averaged incidence (per fish) of voxels for each metric across poles (AP-PP axis). C, Stacked histogram of the relative proportions of the defined classes within each age/condition group. D, Stacked histogram of the relative proportions of defined classes relative to the visually responsive voxels. Scale bars, $20 \mu \mathrm{m}$. Color coding in all cases, same as in $\boldsymbol{A}$.

ing retinal circuits as they appear to define multiple distinct laminae that differentially diminish with increasing age (3-10 dpf). Thus, although dark rearing appeared to have no affect on this functional class of responses as a whole, more subtle effects within individual subtypes of retinal circuits pooled within this functional class cannot be excluded.

In higher vertebrates, the laminar organization within the retinal inner plexiform layer and primary retino-recipient fields (superior colliculus, the mammalian homolog of the tectum) appears to be differentially affected by coordinated activity (Xu and Tian, 2007; Huberman et al., 2009; Kim et al., 2010; Huberman and Niell, 2011). In contrast, a subset of larval zebrafish RGCs (Nevin et al., 2008) exhibited no apparent alterations to their collective axonal lamination within the tectum or their den- dritic organization within the inner plexiform layer, a finding that highlighted the apparent hardwiring of synaptic layers in the zebrafish. Our functionally identified subset of retinal inputs (OS-RGC subtypes), however, do appear to exhibit a dependence on visual drive for their correct lamina placement and within lamina distribution. Indeed, further, their dynamic functional characteristics appear to be driven by visual experience during development, which may also suggest a refinement of the underlying circuits within the retina. Given the relatively sparse incidence of OS voxels and the subtle deficits in laminar organization, only data pooled from several subjects in the form of cumulative histograms of preferred orientation and group parametric maps of spatial organization could reveal such a deficit. It is therefore not surprising that such activity-dependent refine- 
ment has not previously been revealed. Our observation that functional RGC classes exhibit heterogeneous laminar dynamics further suggests that zebrafish are more akin to higher vertebrates than previously thought (Nevin et al., 2008; Kim et al., 2010). Although it should be noted that the OS-RGC functional class of response constitutes only a small fraction of visually responsive voxels within the tectum, the majority of visual circuits within the zebrafish may indeed be hardwired. Further, our findings do not have the scope to comment on whether such alterations in lamination after dark rearing constitutes a failure of molecular guidance cues or a nascent activity refinement. Dark rearing may just alter the relative timings of OS-RGC subtype innervating the tectum, impacting on the specificity of molecular guidance cues that define the correct lamina placement. Further work is required to determine whether fish and mammals occupy a continuum of vertebrate visual development (molecular guidance and activity refinement) or unique evolutionary solutions to laminar construction and organization. This is an important area of research as it will undoubtedly impact on the form and function of visual computations within these different animals.

DS and OS cells are present not only in the retina but in several downstream visual centers: tectal neurons of zebrafish larvae (Niell and Smith, 2005; Gabriel et al., 2012; Grama and Engert, 2012; Hunter et al., 2013), the mouse visual thalamus and superior colliculus (Marshel et al., 2012; Piscopo et al., 2013), and in carnivore and rodent visual cortex (Chapman and Stryker, 1993; Wang et al., 2010; Rochefort et al., 2011). From this wider perspective, the developmental dynamics of DS and OS cells appear diverse and contextual, being dependent on the location within the visual processing stream and the species. For example, in the ferret, orientation selectivity in V1 is initiated without visual experience being present at eye opening (White and Fitzpatrick, 2007). However, direction selectivity is absent at eye opening, requiring visual experience for its subsequent emergence (Li et al., 2006). In comparison, selective neurons in mouse V1 are both evident at eye opening, although only orientation selectivity appears to require ongoing visual experience (Rochefort et al., 2011). Indeed, the mouse also possesses both DS and OS cell types within the visual thalamus (Marshel et al., 2012; Piscopo et al., 2013) and DS-RGC inputs (Huberman et al., 2009; Kay et al., 2011; Rivlin-Etzion et al., 2011). The only across species constant appears to be the activity-independent development of DS-RGCs (Vaney et al., 2012). Indeed, our findings for zebrafish RGCs concur for direction selectivity in this regard, whereas orientation selectivity appears congruent with V1 cortical cells in that they are initiated without visual experience but their subsequent refinement requires activity. Collectively, such developmental diversity poses interesting questions about visual processing across species. In particular, how do population codes within direction space and orientation space compare across species, and how diverse are the circuits that use such representations? Our data may contribute to this debate in two regards, posing intriguing questions for future dissection. First, zebrafish larvae exhibit DSRGC subtypes that appear homologous, in terms of their number and triangular tiling of direction space, to the mouse ON-DSRGCs that project to the accessory optic system (Yonehara et al., 2008; Vaney et al., 2012). Further, zebrafish appear to lack the four cardinally arranged ON-OFF-DS-RGCs that innervate the visual thalamus and superior colliculus in the mouse (Huberman et al., 2009; Kay et al., 2011; Rivlin-Etzion et al., 2011), although a cardinally arranged population code is evident in tectal cells (Hunter et al., 2013). Are the zebrafish DS subtypes homologs of the mammalian ON-DS-RGCs or do they represent a partial rep- resentation of the ON-OFF-DS-RGCs that are expanded into a cardinal population code by local circuits in the tectum? Second, we have isolated four subtypes of OS responses in the zebrafish that collectively represent population codes of orientation space. Indeed, the bilaminar distribution and triangular tiling of orientation space with an over-representation in the vertical axis suggest that two distinct population codes for orientation selectivity in zebrafish RGCs may exist. Do the two vertical subtypes, with different developmental dynamics, have different mechanisms underlying the emergence of orientation selectivity? And is their developmental diversity also reflected in their different roles in visual processing?

Our conceptual approach was to label all RGC axon terminals with a functional indicator of activity and quantitatively characterize coherent response properties to different visual stimuli. Its application in the zebrafish has enabled a quick yet robust assessment of multiple functional populations of responses. Our systems-based approach, revealing coherent population codes, will be a powerful experimental framework with which to probe general rules of construction and computation associated with vertebrate visual processing.

\section{References}

Ahrens MB, Li JM, Orger MB, Robson DN, Schier AF, Engert F, Portugues R (2012) Brain-wide neuronal dynamics during motor adaptation in zebrafish. Nature 485:471-477. CrossRef Medline

Alivisatos AP, Chun M, Church GM, Greenspan RJ, Roukes ML, Yuste R (2012) The Brain Activity Map Project and the challenge of functional connectomics. Neuron 74:970-974. CrossRef Medline

Ben Fredj N, Hammond S, Otsuna H, Chien CB, Burrone J, Meyer MP (2010) Synaptic activity and activity-dependent competition regulates axon arbor maturation, growth arrest, and territory in the retinotectal projection. J Neurosci 30:10939-10951. CrossRef Medline

Bilotta J, Abramov I (1989) Orientation and direction tuning of goldfish ganglion cells. Vis Neurosci 2:3-13. CrossRef Medline

Carandini M (2012) From circuits to behavior: a bridge too far? Nat Neurosci 15:507-509. CrossRef Medline

Chan YC, Chiao CC (2008) Effect of visual experience on the maturation of ON-OFF direction selective ganglion cells in the rabbit retina. Vision Res 48:2466-2475. CrossRef Medline

Chapman B, Stryker MP (1993) Development of orientation selectivity in ferret visual cortex and effects of deprivation. J Neurosci 13:5251-5262. Medline

Easter SS Jr, Nicola GN (1996) The development of vision in the zebrafish (Danio rerio). Dev Biol 180:646-663. CrossRef Medline

Elstrott J, Anishchenko A, Greschner M, Sher A, Litke AM, Chichilnisky EJ, Feller MB (2008) Direction selectivity in the retina is established independent of visual experience and cholinergic retinal waves. Neuron 58 : 499-506. CrossRef Medline

Engert F (2013) Fish in the matrix: motor learning in a virtual world. Front Neural Circuits 6:125. CrossRef Medline

Engert F, Tao HW, Zhang LI, Poo MM (2002) Moving visual stimuli rapidly induce direction sensitivity of developing tectal neurons. Nature 419: 470-475. CrossRef Medline

Engeszer RE, Barbiano LA, Ryan MJ, Parichy DM (2007) Timing and plasticity of shoaling behaviour in the zebrafish, Danio rerio. Anim Behav 74:1269-1275. CrossRef Medline

Fero K, Yokogawa T, Burgess HA, Kalueff A (2011) The behavioral repertoire of larval zebrafish. Neuromethods 52:249-292. CrossRef

Gabriel JP, Trivedi CA, Maurer CM, Ryu S, Bollmann JH (2012) Layerspecific targeting of direction-selective neurons in the zebrafish optic tectum. Neuron 76:1147-1160. CrossRef Medline

Gahtan E, Tanger P, Baier H (2005) Visual prey capture in larval zebrafish is controlled by identified reticulospinal neurons downstream of the tectum. J Neurosci 25:9294-9303. CrossRef Medline

Grama A, Engert F (2012) Direction selectivity in the larval zebrafish tectum is mediated by asymmetric inhibition. Front Neural Circuits 6:59. CrossRef Medline 
Huberman AD, Niell CM (2011) What can mice tell us about how vision works? Trends Neurosci 34:464-473. CrossRef Medline

Huberman AD, Wei W, Elstrott J, Stafford BK, Feller MB, Barres BA (2009) Genetic identification of an On-Off direction-selective retinal ganglion cell subtype reveals a layer-specific subcortical map of posterior motion. Neuron 62:327-334. CrossRef Medline

Hunter P, Lowe A, Thompson I, Meyer M (2013) Emergent properties of the optic tectum revealed by population analysis of direction and orientation selectivity. J Neurosci 33:13940-13945.

Kay JN, De la Huerta I, Kim IJ, Zhang Y, Yamagata M, Chu MW, Meister M, Sanes JR (2011) Retinal ganglion cells with distinct directional preferences differ in molecular identity, structure, and central projections. J Neurosci 31:7753-7762. CrossRef Medline

Kim IJ, Zhang Y, Meister M, Sanes JR (2010) Laminar restriction of retinal ganglion cell dendrites and axons: subtype-specific developmental patterns revealed with transgenic markers. J Neurosci 30:1452-1462. CrossRef Medline

Kimmel CB, Patterson J, Kimmel RO (1974) The development and behavioral characteristics of the startle response in the zebra fish. Dev Psychobiol 7:47-60. CrossRef Medline

Kimmel CB, Ballard WW, Kimmel SR, Ullmann B, Schilling TF (1995) Stages of embryonic development of the zebrafish. Dev Dyn 203:253-310. CrossRef Medline

Levick WR (1967) Receptive fields and trigger features of ganglion cells in the visual streak of the rabbits retina. J Physiol 188:285-307. Medline

Li Y, Fitzpatrick D, White LE (2006) The development of direction selectivity in ferret visual cortex requires early visual experience. Nat Neurosci 9:676-681. CrossRef Medline

Lister JA, Robertson CP, Lepage T, Johnson SL, Raible DW (1999) nacre encodes a zebrafish microphthalmia-related protein that regulates neural-crest-derived pigment cell fate. Development 126:3757-3767. Medline

Marshel JH, Kaye AP, Nauhaus I, Callaway EM (2012) Anterior-posterior direction opponency in the superficial mouse lateral geniculate nucleus. Neuron 76:713-720. CrossRef Medline

Meyer MP, Smith SJ (2006) Evidence from in vivo imaging that synaptogenesis guides the growth and branching of axonal arbors by two distinct mechanisms. J Neurosci 26:3604-3614. CrossRef Medline

Mumm JS, Williams PR, Godinho L, Koerber A, Pittman AJ, Roeser T, Chien $\mathrm{CB}$, Baier H, Wong RO (2006) In vivo imaging reveals dendritic targeting of laminated afferents by zebrafish retinal ganglion cells. Neuron 52: 609-621. CrossRef Medline

Nevin LM, Taylor MR, Baier H (2008) Hardwiring of fine synaptic layers in the zebrafish visual pathway. Neural Dev 3:36. CrossRef Medline

Niell CM, Smith SJ (2005) Functional imaging reveals rapid development of visual response properties in the zebrafish tectum. Neuron 45:941-951. CrossRef Medline

Niell CM, Stryker MP (2008) Highly selective receptive fields in mouse visual cortex. J Neurosci 28:7520-7536. CrossRef Medline

Niell CM, Meyer MP, Smith SJ (2004) In vivo imaging of synapse formation on a growing dendritic arbor. Nat Neurosci 7:254-260. CrossRef Medline

Nikolaou N, Lowe AS, Walker AS, Abbas F, Hunter PR, Thompson ID, Meyer MP (2012) Parametric functional maps of visual inputs to the tectum. Neuron 76:317-324. CrossRef Medline

Ourselin S, Roche A, Subsol G, Pennec X, Ayache N (2001) Reconstructing a 3D structure from serial histological sections. Image Vision Computing 19:25-31. CrossRef
Piscopo DM, El-Danaf RN, Huberman AD, Niell CM (2013) Diverse visual features encoded in mouse lateral geniculate nucleus. J Neurosci 33:4642-4656. CrossRef Medline

Quiroga RQ, Nadasdy Z, Ben-Shaul Y (2004) Unsupervised spike detection and sorting with wavelets and superparamagnetic clustering. Neural Comput 16:1661-1687. CrossRef Medline

Ramdya P, Engert F (2008) Emergence of binocular functional properties in a monocular neural circuit. Nat Neurosci 11:1083-1090. CrossRef Medline

Rivlin-Etzion M, Zhou K, Wei W, Elstrott J, Nguyen PL, Barres BA, Huberman AD, Feller MB (2011) Transgenic mice reveal unexpected diversity of on-off direction-selective retinal ganglion cell subtypes and brain structures involved in motion processing. J Neurosci 31:8760-8769. CrossRef Medline

Robles E, Filosa A, Baier H (2013) Precise lamination of retinal axons generates multiple parallel input pathways in the tectum. J Neurosci 33:50275039. CrossRef Medline

Rochefort NL, Narushima M, Grienberger C, Marandi N, Hill DN, Konnerth A (2011) Development of direction selectivity in mouse cortical neurons. Neuron 71:425-432. CrossRef Medline

Schroeter EH, Wong RO, Gregg RG (2006) In vivo development of retinal ON-bipolar cell axonal terminals visualized in nyx::MYFP transgenic zebrafish. Vis Neurosci 23:833-843. CrossRef Medline

Sernagor E, Grzywacz NM (1995) Emergence of complex receptive field properties of ganglion cells in the developing turtle retina. J Neurophysiol 73:1355-1364. Medline

Swindale NV (1998) Orientation tuning curves: empirical description and estimation of parameters. Biol Cybern 78:45-56. CrossRef Medline

Vaney DI, Sivyer B, Taylor WR (2012) Direction selectivity in the retina: symmetry and asymmetry in structure and function. Nat Rev Neurosci 13:194-208. CrossRef Medline

Venkataramani S, Taylor WR (2010) Orientation selectivity in rabbit retinal ganglion cells is mediated by presynaptic inhibition. J Neurosci 30: 15664-15676. CrossRef Medline

Wang L, Sarnaik R, Rangarajan K, Liu X, Cang J (2010) Visual receptive field properties of neurons in the superficial superior colliculus of the mouse. J Neurosci 30:16573-16584. CrossRef Medline

White LE, Fitzpatrick D (2007) Vision and cortical map development. Neuron 56:327-338. CrossRef Medline

Wyart C, Del Bene F (2011) Let there be light: zebrafish neurobiology and the optogenetic revolution. Rev Neurosci 22:121-130. CrossRef Medline

Xiao T, Baier H (2007) Lamina-specific axonal projections in the zebrafish tectum require the type IV collagen Dragnet. Nat Neurosci 10:1529-1537. CrossRef Medline

Xu HP, Tian N (2007) Retinal ganglion cell dendrites undergo a visual activity-dependent redistribution after eye opening. J Comp Neurol 503: 244-259. CrossRef Medline

Yonehara K, Shintani T, Suzuki R, Sakuta H, Takeuchi Y, NakamuraYonehara K, Noda M (2008) Expression of SPIG1 reveals development of a retinal ganglion cell subtype projecting to the medial terminal nucleus in the mouse. PloS One 3:e1533. CrossRef Medline

Yonehara K, Ishikane H, Sakuta H, Shintani T, Nakamura-Yonehara K, Kamiji NL, Usui S, Noda M (2009) Identification of retinal ganglion cells and their projections involved in central transmission of information about upward and downward image motion. PloS One 4:e4320. CrossRef Medline 